\title{
Atomic Structures and Properties of Ceramic Interfaces - Combination of Cs-corrected STEM and First Principles Calculations
}

\author{
Y.Ikuhara ${ }^{1,2,3}$, Y.Sato ${ }^{1}$, S.D. Findlay ${ }^{1}$,T.Mizoguchi ${ }^{1}$, N.Shibata ${ }^{1}$ and T.Yamamoto ${ }^{1,2}$
}

1. Institute of Engineering Innovation, the University of Tokyo, Tokyo 116-0013, Japan

2. Nanostructures Research Laboratory, Japan Fine Ceramics Center, Nagoya, 456-8587, Japan

3. WPI Advanced Institute for Materials Research, Tohoku University, Sendai, 980-8577, Japan

Grain boundaries and interfaces of crystals have peculiar electronic structures, caused by the disorder in periodicity, providing the functional properties, which cannot be observed in a perfect crystal. In the vicinity of the grain boundaries and interfaces around the order of $1 \mathrm{~nm}$, dopants or impurities are often segregated, and they play a crucial role in the material properties. We call these dopants "function providing elements", which have the characteristics to change the macroscopic properties of the materials drastically. In this study, we focus our attention on grain boundaries and interfaces of various ceramics, to which "function providing elements" are doped, and introduce the latest results of the microstructure analyzed in detail by Cs-corrected STEM. Furthermore, the mechanism of the "function providing elements" are analyzed by the first principles calculation based on these observation results. Fig.1(a) shows a typical high-angle annular dark-field (HAADF) STEM image of the Pr-doped ZnO 549 GB[1]. Viewed from [0001] projection, $\mathrm{Zn}$ and $\mathrm{O}$ are aligned in the same atomic columns. Positions of the $\mathrm{Zn}-\mathrm{O}$ columns appear as bright spots because of the incoherent nature of HAADF STEM imaging. Much brighter spots indicate the presence of heavier atoms than $\mathrm{Zn}(Z=30)$, in this case $\operatorname{Pr}$ $(Z=59)$. EELS taken from the GB also show the presence of $\operatorname{Pr}$, and analysis of the spectra indicates that $\operatorname{Pr}$ is in the $3+$ state. It is found that Pr segregates at the specific atomic columns of the GB, and there is a unique periodicity for the atomic location of Pr. From the left to the right, Pr atoms locate at one and two atomic columns alternatively along the GB plane. This unique configuration of Pr is closely related to the GB atomic structures. The HAADF STEM image was compared with the theoretically obtained stable atomic structure of undoped $\mathrm{ZnO}$ GB in Fig. 1(b). Zn-O columns of the undoped $\mathrm{ZnO}$ GB match with bright spots of the HAADF STEM image. This suggests that framework structure of the GB is not significantly influenced by the Pr-doping, and Pr substitute $\mathrm{Zn}$ sites in the atomic columns. Considering the periodicity along the GB, Pr segregate at three kinds of atomic columns of the GB. Fig 2 shows the HAADF STEM images of $\mathrm{Y}$ doped $\mathrm{Al}_{2} \mathrm{O}_{3} \Sigma 13$ grain boundary, which were observed from three directions, one plan view and two cross sectional directions [2]. In the plan view image, image filtering was applied to remove the background and improve the visibility of the $\mathrm{Y}$ atom arrangement. The two-dimensional ordered array of individual $\mathrm{Y}$ atoms is clearly evident. The $\mathrm{Y}$ atom array along the $<1 \overline{2} 10>$ and $<\overline{2} 021>$ directions in the plan view image corresponds well to 
the $\mathrm{Y}$ atomic column positions observed in the two cross-sectional images. In addition, there are some clear deviations of the $\mathrm{Y}$ atom positioning from the ordered positions. As can be seen in the figure, some $\mathrm{Y}$ atoms are shifted in between the ordered array sites, as indicated by the arrows. This demonstrate that $\mathrm{Y}$ positioning at the grain boundary is basically ordered in two dimensions based on their specific stable atom positions on the interface, but that some fluctuation or disordering exists, perhaps due to trapping and/or overflowing onto the metastable sites. It can be thus said that planview HAADF STEM can be a very powerful method for directly imaging individual atoms within materials, bringing us a crucial step towards the full three-dimensional characterization of interface atomic structures.

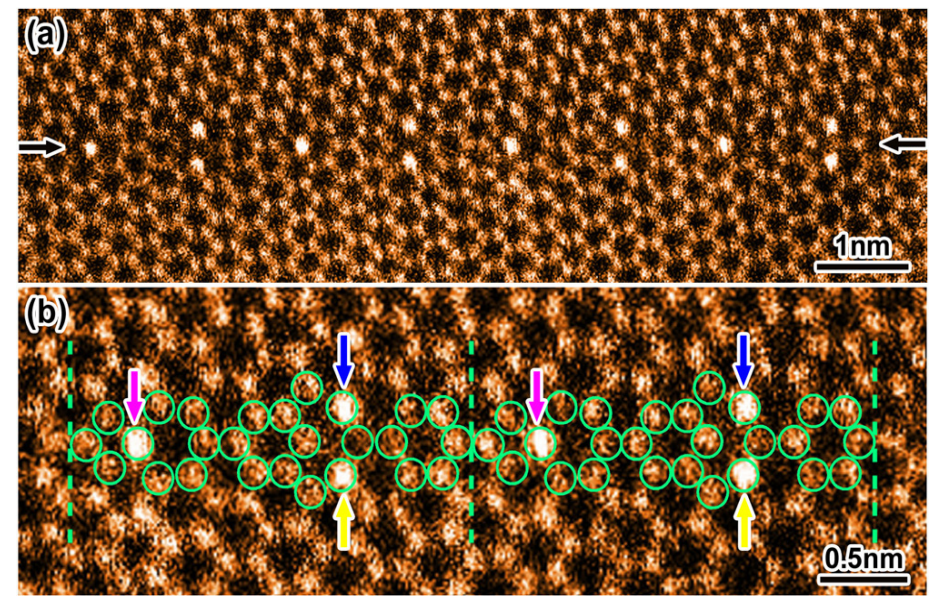

Fig.1 (a) HAADF STEM image of a Pr-doped $\mathrm{ZnO} \Sigma 49$ GB. Incident beam direction and GB plane are parallel to [0001] and $(35 \overline{8} 0)$ of both crystals, respectively. Arrows show the position of GB plane. (b) Enlarged image with the framework structure of undoped $\mathrm{ZnO} \mathrm{GB}$, which is superimposed by green circles. Dotted lines show the periodicity along the GB plane. Colored arrows indicate the location of $\mathrm{Pr}$ atoms, in which three different colors show three different GB atomic sites.

\section{References}

[1] N. Shibata, S.D. Findlay, S. Azuma, T. Mizoguchi, T. Yamamoto and Y. Ikuhara, Nature Materials, 8 , 654-658 (2009).

[2]Y. Sato, T. Mizoguchi, N. Shibata, T. Yamamoto,

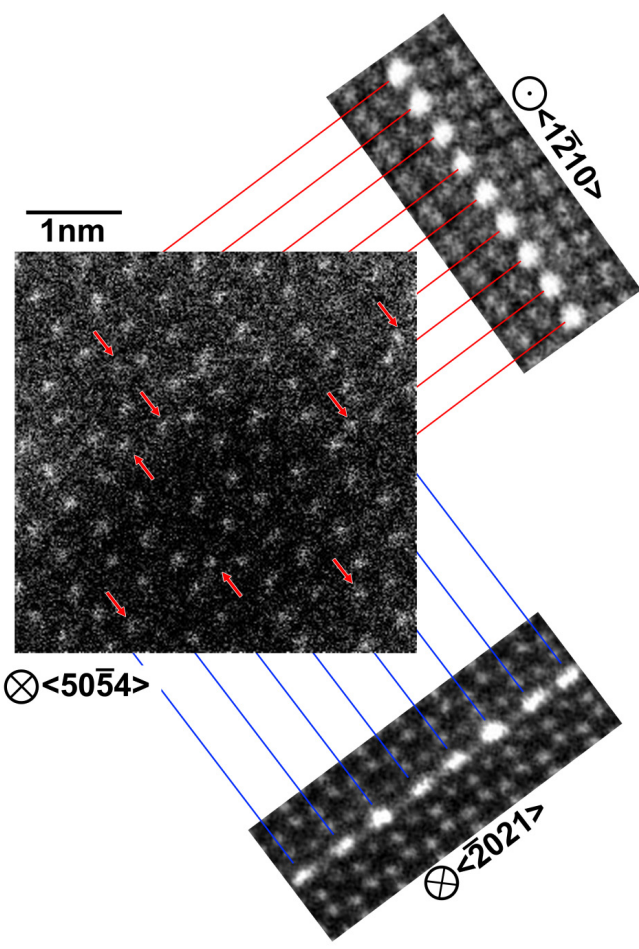

Fig.2. Three dimensional HAADF STEM observations, showing a plan-view and two cross-sectional images in $\mathrm{Y}$ doped $\mathrm{Al}_{2} \mathrm{O}_{3} \Sigma 13$ grain boundary. The image is displayed with a nonlinear intensity scale to highlight bright features. The two-dimensional ordered array of individual $\mathrm{Y}$ atoms is clearly observed.

T. Hirayama and Y. Ikuhara, Phys. Rev. B ,80, 094114 (2009).

[3]Part of this work was supported by the Grants-in-Aid for Scientific Research on Priority Areas "Nano Materials Science for Atomic-scale Modification" (No.19053001) from the Ministry of Education, Culture, Sports, Science and Technology (MEXT). 